\title{
INTUMESCENCES
}

Société Hydrotechnique de France

15 juin 1961

\section{Application du calcul des intumescences sur machine électronique à divers cas pratiques}

\section{Application of translation wave calculations on an electronic computer to practical cases}

\author{
PAR
}

A. PREISSMANN

ET

G. WERNER

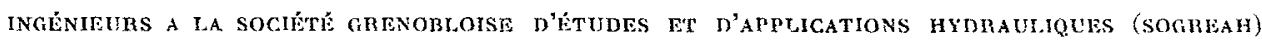

\begin{abstract}
Discussion de trois exemples d'application possible du calcul des intumescences sur machine électronique :

1. Essais systématiques de calcul d'ondes de crue en vile d'estimer la validité de certaines règles semi-empiriques sur la propagation des crues;

2. Etude de la stabilité du réglage de vannes automatiques;

3. Calcul de l'écoulement dans une galerie qui se trouve tantôt en charge, tantòt à surface libre.
\end{abstract}

\begin{abstract}
The three following possible applicalions of electronic computer to solitary wave calculations are discussed:

1. Systematic trial flood wave calculations, in order to assess the validity of certain semiempirical rules governing flood propagation;

2. Study of the stability of control afforded b!l antomatic gates;

3. Calculation of flows in a tunnel allernaling between pressure flow and free surface con-
\end{abstract} ditions.

\section{I. - INTRODUCTION}

Depuis lapparilion des machines à calculer ćlectroniques, divers organismes les ont utilisées avec succès pour traiter les problèmes de propagation d'intumescences dans les canaux et rivières. L'expérience, encore limitée, de la SOGREAH à ce sujet, nous permet d'affirmer que les problèmes en question sont bien adaptés aux machines électroniques, vu que le calcul consiste à répéter un très grand nombre de fois les mêmes opérations. Toutefois, avec une machine du type IBM 650 dont dispose la SOGREAH, le temps de calcul d'une crue est relativement long. Dans ces conditions, les machines les plus rapides sont les plus économiques.

Nous ne pensons pas, d'ailleurs, qu'il soit nécessaire de traiter chaque cas d'espèce sur machine électronique; dans certains cas, les règles semi-empiriques utilisées dans la prévi- sion des crues permettent une évaluation rapide de l'évolution de la crue. Il est donc intéressant d'étudier la validité et les limitations des systèmes pratiques de prévision des crues.

Dans une telle étude, les machines électroniques peuvent rendre de grands services; elles permettent de comparer les résultats d'un calcul sommaire et d'un calcul plus précis pour différents types de crues et pour différents types de rivières. C'est de cela que traite la première partie de l'exposé.

Dans une deuxième partie, nous discuterons de l'utilisation des machines électroniques dans un problème de régulation de vannes automatiques.

Enfin, nous montrerons comment les programmes de calcul des intumescences en canal peuvent également être utilisés dans les systèmes de conduites, tantôt à surface libre, tantôt en charge. 


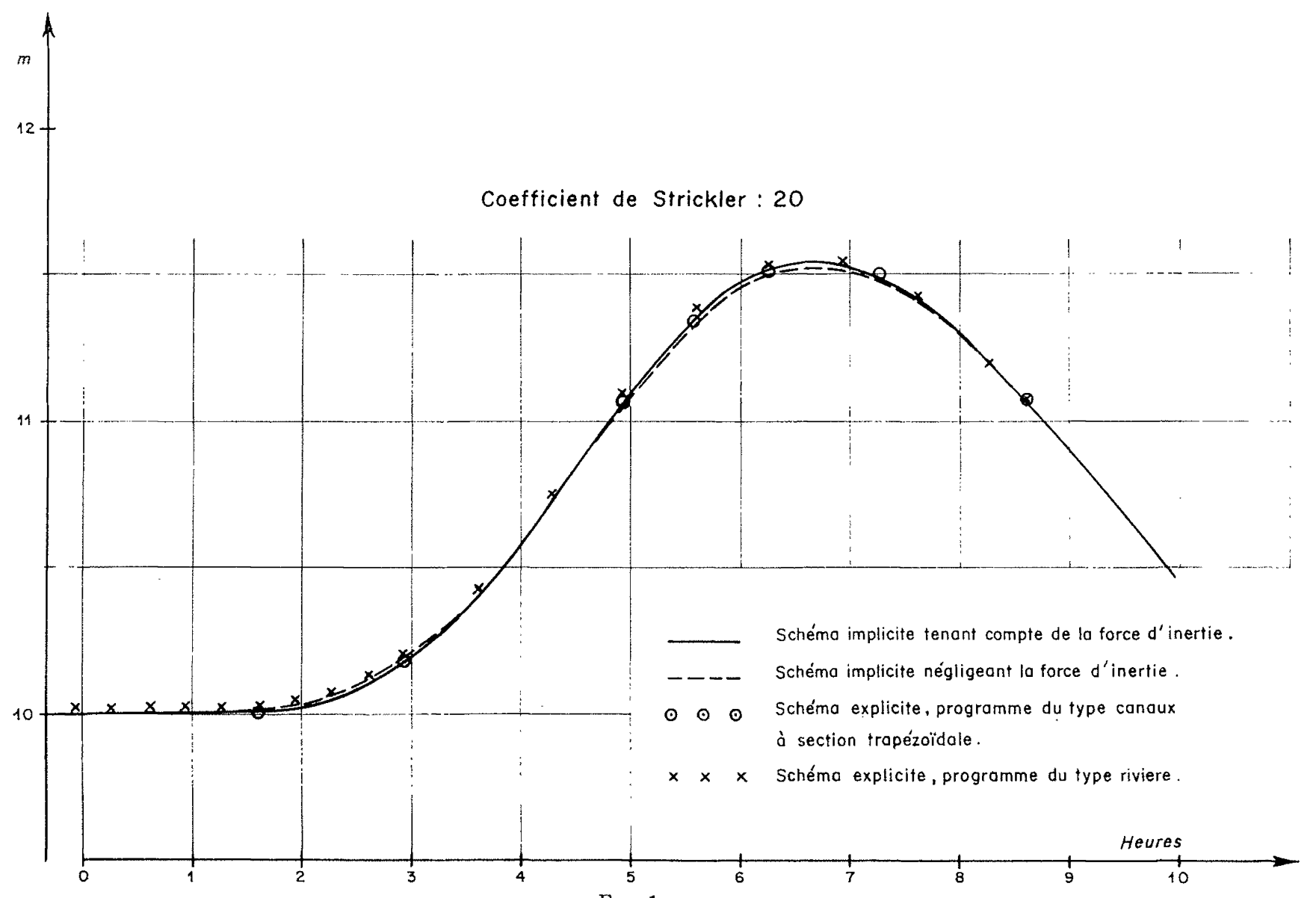

Niveaux dans la section aval du canal trapézoïdal calculés à l'aide de méthodes différentes.

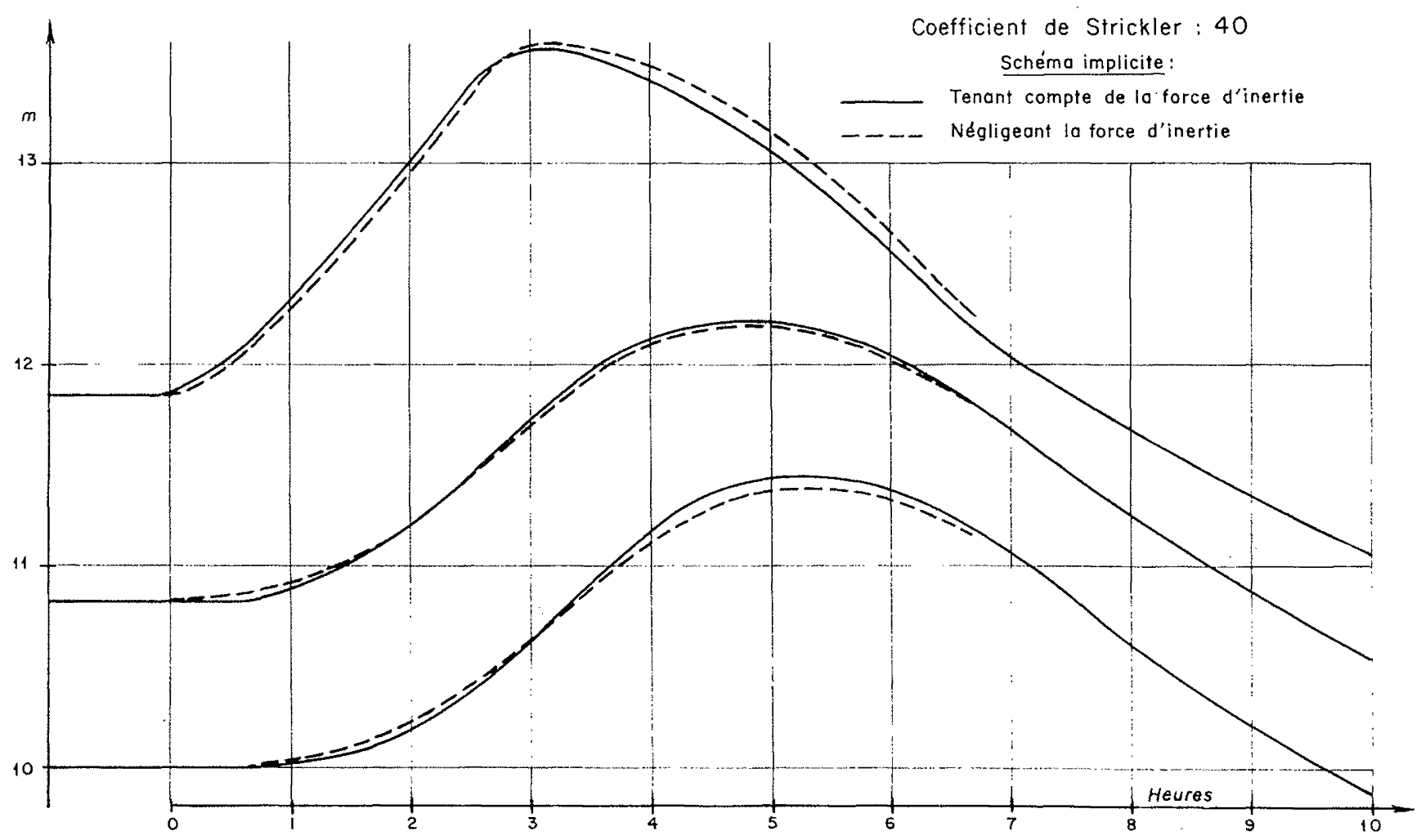

Fig. 2

Niveaux dans un canal trapézoïdal calculés dans trois sections en amont, à mi-longueur et en aval. 


\section{II. - EXAMEN SYSTÉMATIQUE DES SIMPLIFICATIONS SUCCESSIVES APPORTÉES AUX EQUATIONS DE L'ÉCOULEMENT NON STATIONNAIRE}

Les équations de l'écoulement non stationnaire, dites de Saint-Venant, s'écrivent :

$$
\begin{gathered}
\frac{\partial(\mathrm{S} v)}{\partial x}+\mathrm{B} \frac{\partial h}{\partial t}=0 \\
\frac{1}{g}\left(\frac{\partial v}{\partial t}+v \frac{\partial v}{\partial x}\right)=i_{0}-\frac{\partial h}{\partial x}-\mathrm{K}|v| v
\end{gathered}
$$

où :

$$
\begin{aligned}
\mathrm{S}(x, h) & =\text { section mouillée } \\
\mathrm{B}(x, h) & =\frac{\partial \mathrm{S}}{\partial h}=\text { largeur } \\
v(x, t) & =\text { vitesse moyenne de l'écoulement } \\
h(x, t) & =\text { profondeur } \\
i_{0}(x) & =\text { pente du fond } \\
\mathrm{K}(x, h) & =\text { coefficient de résistance }
\end{aligned}
$$

La première des équations, qui exprime la conservation des volumes d'eau, n'est en général pas simplifiée. Dans l'équation dynamique (2) par contre, il apparaît souvent que le terme de gauche (terme d'inertie), qui représente la pente due à l'accélération de l'eau, est faible par rapport à chacun des termes de droite. Cette circonstance se présente en particulier lors de la propagation d'ondes de crue naturelles assez lentes dans les rivières présentant une pente appréciable. La suppression du terme de gauche, n'est, par contre, pas licite lorsque la pente de la rivière est faible (par exemple dans les estuaires) ou dans les canaux d'usines hydroélectriques lorsque les manœuvres des usines ou des barrages sont rapides.

Il faut noter que la suppression du terme d'inertie modifie profondément les propriétés mathématiques du système (1), (2). En effet, alors que le système complet est hyperbolique, le système simplifié est parabolique. Cela se marque dans les conditions qui définissent le problème de la détermination de $h$ et $v$.

Dans le système complet, il faut se donner :

- une condition à l'amont et une condition à l'aval du tronçon considéré (conditions aux limites);

- les valeurs de $h$ et de $v$ en tout point du tronçon à l'instant du départ du calcul (conditions initiales).

Dans le système simplifié, les conditions aux limites sont les mêmes, mais, dans les conditions initiales, on ne donne que les valeurs de $h$, les valeurs de $v$ se déduisant de la relation :

$$
i_{0}-\frac{\partial h}{\partial x}-\mathrm{K}|v| v=0
$$

Afin de nous rendre compte de l'influence du terme d'inertie sur la propagation des crues, nous avons traité l'onde de crue dans un canal trapézoïdal de $40 \mathrm{~km}$ de long. Les conditions aux limites sont les suivantes :

- à l'aval, on se donne une loi hauteur/débit;

- à l'amont, on injecte un débit passant, en 160 minutes, de $800 \mathrm{~m}^{3} / \mathrm{s}$ à $1600 \mathrm{~m}^{3} / \mathrm{s}$, puis de $1600 \mathrm{~m}^{3} / \mathrm{s}$ à $400 \mathrm{~m}^{3} / \mathrm{s}$ en 240 minutes.

La condition initiale correspond à l'écoulement uniforme de $800 \mathrm{~m}^{3} / \mathrm{s}$. Les calculs ont été faits pour trois coefficients de rugosité de Strickler :

$$
k=20, \quad k=40, \quad k=80 \mathrm{~m}^{1 / 3} / \mathrm{s}
$$

La comparaison des calculs effectués en posant le terme d'inertie égal à zéro avec les calculs complets montrent que, pour $k=20$, l'influence du terme d'inertie est négligeable; pour $k=40$, l'évolution des niveaux est légèrement modifiée; pour $k=80$, il est nécessaire d'introduire le terme d'inertie (voir fig. 1, 2, 3).

Des essais de calcul de ce type ont pour but essentiel de déceler les cas dans lesquels l'inertie joue un rôle négligeable. En effet, le programme de calcul se simplifie considérablement si l'on peut poser le terme d'inertie égal à zéro, et le calcul effectué dans un cas concret est sensiblement plus court.

Dans certains cas, la simplification des équations (1), (2) peut se poursuivre. En effet, lors des crues lentes, la quantité $(\partial h / \partial x)$ est faible par rapport aux termes $i_{0}$ et $\mathrm{K}|v| v$. Cela revient $\dot{a}$ admettre que les courbes hauteur/débit univoques valables en régime permanent s'appliquent encore au régime non stationnaire. On écrira donc que, pour chaque section $\mathrm{X}$ :

$$
\mathrm{Q}_{x}=v_{x} \mathrm{~S}_{x}=\mathbf{Q}_{x}(h)
$$

De même, naturellement $\mathrm{S}$, la section mouillée, est une fonction univoque de la cote de la surface donnée par $h, \mathrm{~S}_{x}=\mathrm{S}_{x}(h)$. Dans ces conditions, l'équation de continuité (1) prend la forme :

$$
\frac{\partial Q}{\partial x}+\frac{\partial Q}{\partial t}\left(\frac{\partial S}{\partial Q}\right)=0
$$




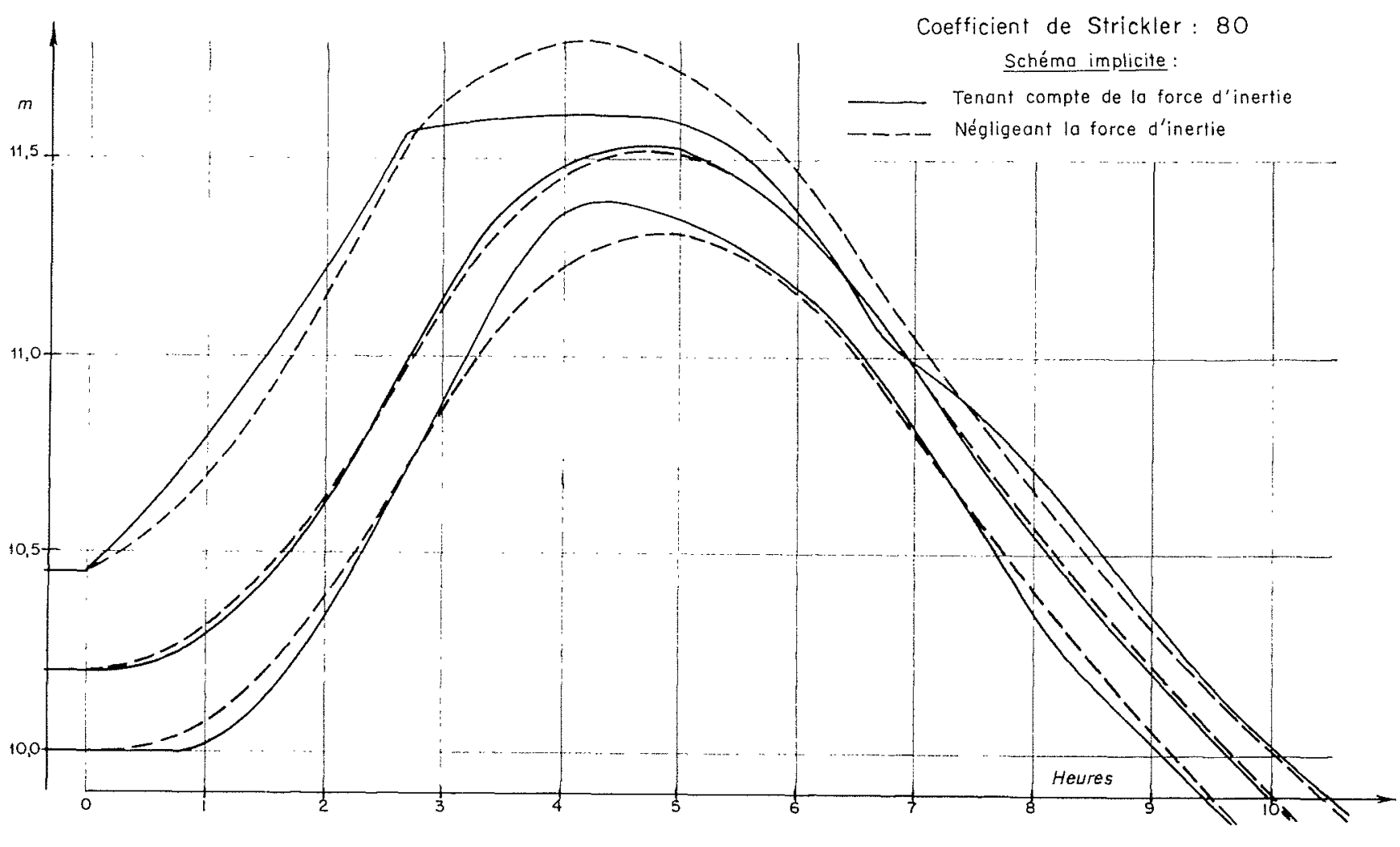

FIG. 3

Niveaux dans un canal trapézoïdal calculés dans trois sections en amont, à mi-longueur ef en aval.

$(\partial \mathrm{S} / \partial \mathrm{Q})_{x}$ représente le rapport entre l'augmentation de la section mouillée et l'augmentation du débit lorsque la cote du niveau varie à la section $X$. L’équation (3) à résoudre se distingue du système (1), (2) ou du système simplifié (1), (2') en ce sens qu'on n'a ici plus qu'une équation aux dérivées partielles du premier degré. Pour définir le problème, il faut se donner :

- la condition initiale $\mathrm{Q}=\mathrm{Q}_{0}(x)$ pour $t=t_{0}$;

- en une seule condition $Q=Q_{1}(t)$ a la limite amont.

De par sa nature, l'équation (3) est impuissante à décrire la propagation des ondes de crues perturbées à l'aval par des ouvrages mobiles; la propagation de l'onde ne se fait que dans une seule direction et ne permet pas de tenir compte de perlurbations venant de l'autre direction.

Lorsqu'il s'agit de petites ondes et que la quantité $(\partial \mathrm{S} / \partial \mathrm{Q})_{x}$ est voisine d'une constante $(1 / c)$, la solution générale de (3) s'écrit :

$$
\mathrm{Q}=\mathrm{Q}(x-c t)
$$

(c est la célérité de l'onde de crue), la détermination de $\mathrm{Q}$ est alors immédiate.

La plupart des systèmes de prévision de crues (en particulier la méthode de concordance des échelles) s'appuient sur des équations du type (3). Il n'est donc pas sans intérêt d'examiner la validité de l'équation (3) dans différents cas pratiques, ce qui permettra de fixer les limites d'application des procédés de prévision et éventuellement de modifier quelque peu ces derniers de façon à les améliorer quelque peu.

En reprenant l'exemple du canal prismatique de $40 \mathrm{~km}$ de long avec $k=40$, nous avons comparé les résultats de l'intégration du système (1), (2) avec celle de l'équation (3).

On remarque que la propagation de la crue est très mal représentée par l'intégration de l'équation (3). En examinant la propagation de la crue, telle qu'elle résulte des équations (1), (2), on constate que la quantité ( $\partial h / \partial x)$ est loin d'être négligeable par rapport à $\dot{i}_{0}$. (Les valeurs absolues des deux quantités sont parfois sensiblement égales.) Nous avons donc choisi un exemple tel que le mauvais accord des denx méthodes de calcul ne faisait aucun doute. Pour réduire l'importance du terme $(\partial h / \partial x)$, on peut modifier les données relatives à la crue de dcux façons différentes:

- soit en diminuant l'intensité de la crue, tout en conservant sa durée. On pourrait, par exemple, passer, à l'amont, de $800 \mathrm{~m}^{3} / \mathrm{s}$ à $900 \mathrm{~m}^{3} / \mathrm{s}$ 
en 160 minutes, puis de $900 \mathrm{~m}^{3} / \mathrm{s}$ à $750 \mathrm{~m}^{3} / \mathrm{s}$ en 240 minutes;

- soit en augmentant la durće de la crue, tout en conservant son intensité. On pourrait ainsi passer de $800 \mathrm{~m}^{3} / \mathrm{s}$ à $1600 \mathrm{~m}^{3} / \mathrm{s}$ en 16 heures, puis de $1600 \mathrm{~m}^{3} / \mathrm{s}$ à $400 \mathrm{~m}^{3} / \mathrm{s}$ en 24 heures.

Dans les deux cas, la valeur de $(\partial h / \partial x)$ serait faible par rapport à $i_{0}$. On constatera cependant, en faisant le calcul, que l'intumescence est mal reproduite dans le premier cas et très sensiblement mienx dans le deuxième.

Afin d'interpréter les résultats et de montrer sur un exemple simple dans quel sens le terme négligé $\partial h / \partial x$ agit, nous avons traité le cas d'une onde de crue faible se superposant à un écoulement uniforme et permanent de débit $\mathrm{Q}_{0}$ dans un canal trapézoïdal. En linéarisant autour de l'état permanent et en éliminant $v$, on obtient à partir de $(1),\left(2^{\prime}\right)$ :

$$
\frac{\partial h}{\partial t}+c_{0} \frac{\partial h}{\partial x}-{\underset{2}{2} i_{0}}_{\mathrm{B}_{0}} \frac{\partial^{2} h}{\partial x^{2}}=0
$$

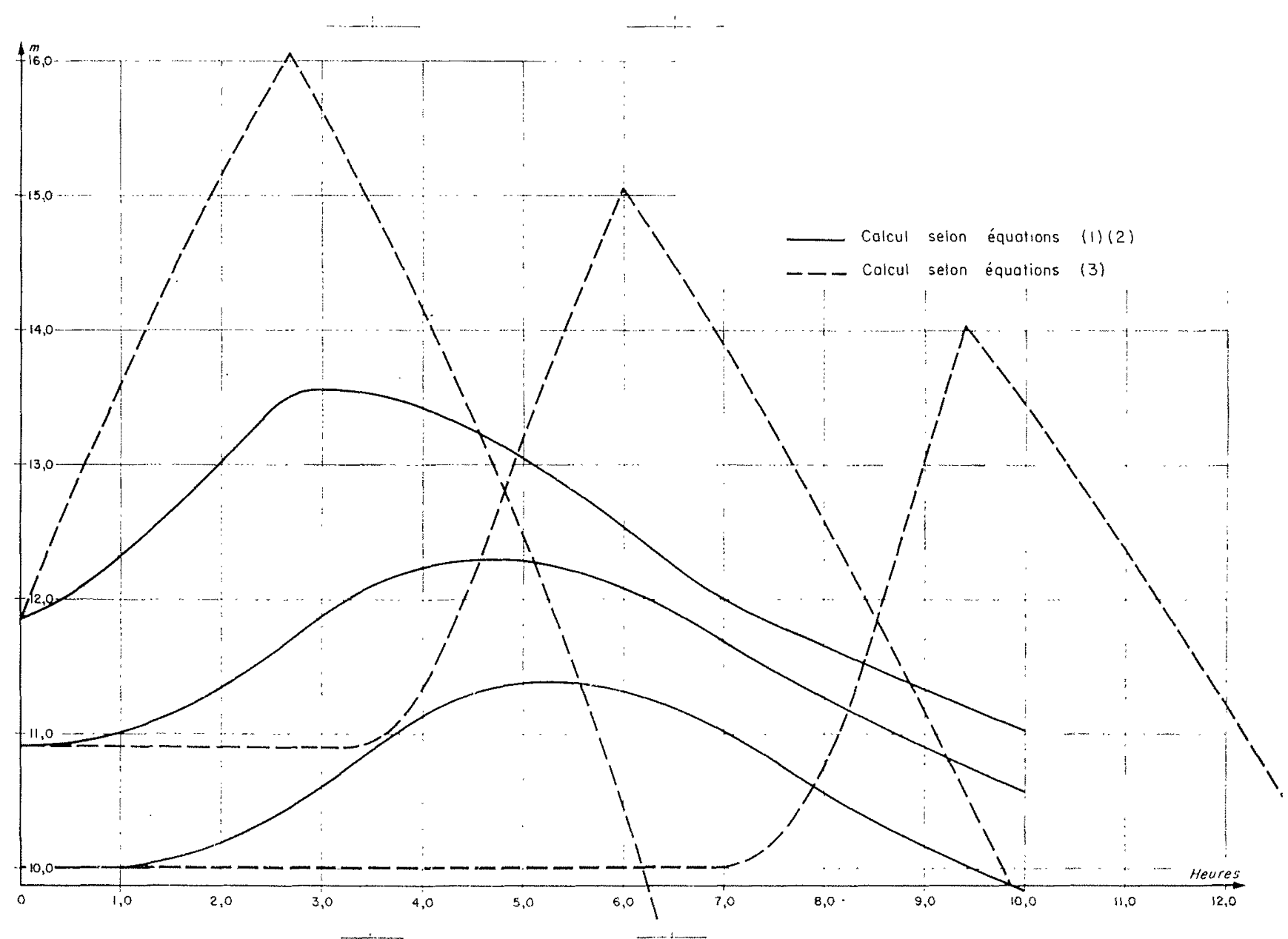

Fig. 4

Niveaux dans un canal trapézoïdal calculés dans trois sections en amołt, à mi-longueur ef en aval au lieu de l'équation linéarisée obtenue à partir de (3)

$$
\frac{\partial h}{\partial t}+c_{0} \frac{\partial h}{\partial x}=0
$$

$c_{0}$ représente la célérité $(\partial Q / \partial S)$ au voisinage de la profondeur de l'écoulement uniforme. L'équation (6) possède la solution générale:

$$
h=h(x, t)=h(x-c t)
$$

L'intumescence se déplace d'un bloc en conservant sa forme. Par contre, l'équation (5) contient un terme impliquant une «diffusion» de l'intumescence. La solution de (5) s'écril, en effet :

$$
h(x, t)=h^{\star}(\xi, t)=h^{\star}\left(x-c_{0} t, t\right)
$$

où la fonction $h^{\star}$ satisfail à l'écuation de la chaleur :

$$
\frac{\partial h^{\star}}{\partial t}=\frac{\mathrm{Q}_{0}}{2 i_{0} \mathrm{~B}_{0}} \frac{\partial^{2} h^{*}}{\partial \xi^{2}}
$$


avec le coefficient de diffusion $\left(Q_{0} / 2 i_{0} B_{0}\right)$, la crue diffuse (s'aplatit) en même temps qu'elle progresse.

Un critère approximatif de validité de l'équation (3) consiste à former le rapport:

$$
\frac{L^{2}}{a^{2} \mathrm{~T}}
$$

où $L$ représente une longueur caractéristique de l'intumescence,

$a^{2}$ le coefficient de diffusion $\frac{Q_{0}}{2 i_{0} B_{0}}$;

$\mathrm{T}$ le «temps de parcours» de l'íntumescence entre l'amont et l'aval, égal à la distance entre l'amont et l'aval divisée par la vitesse $c_{0}$. Si le rapport est nettement supérieur à 1 , l'équation (3) donne une approximation acceptable. Dans le cas traité, le rapport est d'environ $1 / 6$. En réduisant l'intensité de la crue, on ne modifie rien au rapport caractéristique.
Naturellement, les considérations qui précèdent ne sont strictement valables que pour de faibles intumescences dans un canal prismatique très long. Cependant, en procédant par analogie dans les cas réels toujours complexes, il sera possible de prévoir par exemple la «durée» d'une crue à partir de laquelle les règles du «flood routing 》 sont pratiquement valables. En effet, lorsque la crue et la décrue sont très lentes, la diffusion est relativement très faible. Eventuellement, les systèmes de prévision peuvent être améliorés par des corrections globales qui devront être testées pour différents types de crues et c'est ici qu'une application des machines électroniques nous semble s'imposer. En effet, à moins de disposer d'observations sûres et nombreuses, la validité des règles de prévision ne sera pas assurée. En reproduisant, sur un modèle mathématique, des crues de types divers, on peut augmenter sensiblement le nombre de «points de mesure».

\section{III. - UTILISATION DES MACHINES ÉLECTRONIQUES DANS L'ETUDE DE LA STABILITE DE VANNES AUTOMATIQUES}

On sait que, dans certains cas, le réglage destiné à maintenir un niveau constant à l'aval de vannes automatiques peut être instable. L'étude de la stabilité s'impose donc. Cette étude doit tenir compte à la fois des caractéristiques du canal amont et des caractéristiques de la régulation du niveau. Par rapport aux problèmes de régulation habituels, la complication réside dans le fait que la « réponse » du canal, régie par une équation aux dérivées partielles, ne s'exprime pas simplement.

$V_{u}$ la complexité du problème, on peut se demander si le procédé le plus adéquat d'étude de stabilité ne devrait pas consister en une reproduction pure et simple par le calcul de l'évolution du système soumis à une légère perturbation initiale. La disparition progressive de la perturbation ou au contraire son amplification constituerait un critère pratique de stabilité.

En principe, le calcul de l'évolution du système par un procédé aux différences finies est naturellement possible. Il suffit d'utiliser un programme de calcul d'ondes dans un canal en prévoyant un programme particulier (intégration d'une équation différentielle ordinaire) pour le mouvement de la vanne à l'aval du canal. Toutefois, on se rend compte facilement que, pour obtenir une précision acceptable, il conviendrait de choisir des intervalles de temps très courts, ce qui conduit à des temps de calcul très apprèciables.

Afin d'étudier la stabilité, nous avons suivi la voie indiquée par Nyquist en linéarisant au préalable, autour de l'état d'équilibre, aussi bien les équations de l'écoulement en canal que les équations du système de régulation. Bien que nous n'ayons introduit aucun élément nouveau, nous présenterons sommairement le principe de la méthode.

Admettons, pour fixer les idées, que le débit d'alimentation à l'amont du canal soit maintenu constant; dans ce cas, une petite perturbation sinusoïdale $h=h_{1} e^{2 \pi i \nu t}$ à l'aval du canal détermine une perturbation sinusoïdale $q=q_{1} e^{2 \pi i y t}$ du débit au même point. Le rapport $\left(q_{1} / h_{1}\right)$ est une quantité complexe qui dépend de la fréquence $v$. Le point représentatif du rapport $\left(q_{1} / h_{1}\right)$ décrit une certaine courbe dans le plan complexe avec $\vee$ comme paramètre (courbe de réponse en fréquence du canal).

De même, lor'squ'on impose au système de réglage une variation sinusoïdale $h_{2} e^{2 \pi i^{\prime \prime t}} \mathrm{du}$ niveau à l'amont de la vanne, il résulte une variation sinusoïdale du débit de $q_{2} e^{2 \pi i v t}$ sous la vanne. La courbe décrite par le point $q_{2} / h_{2}$ dans le plan complexe constitue la courbe de réponse en fréquence du système de réglage.

En fait, $h_{1}$ et $h_{2}$ ont la même signification (le 
niveau aval du canal est le niveau amont de la vanne); de même pour $q_{1}$ et $q_{2}$ (le débit aval du canal est égal au débit sous la vanne).

Si une oscillation entretenue sinusoïdale $\mathrm{du}$ système dans son ensemble devait être possible, on devrait avoir pour une certaine valeur de $v$ :

$$
\frac{q_{2}}{h_{2}}=\frac{q_{1}}{h_{1}}
$$

dans ce cas, la courbe représentative de la quantité

$$
\frac{q_{2}}{h_{2}}: \frac{q_{1}}{h_{1}}
$$

passerait par le point $(+1,0)$ du plan complexe.
Dans le cas général, on construit la courbe :

$$
\frac{q_{2}}{h_{2}}: \frac{q_{1}}{h_{1}}
$$

dans le plan complexe avec y comme paramètre. Le critère de stabilité de Nyquist s'énonce alors ainsi :

Si la courbe représentative de $\frac{q_{2}}{h_{2}}: \frac{q_{1}}{h_{1}}$ parcourue dans le sens des $v$ croissants ne contourne jamais le point $(+1,0)$ en le laissant à droite, alors le système est stable.

Nous avons effectué des calculs dans un cas relativement simple et nous avons obtenu les

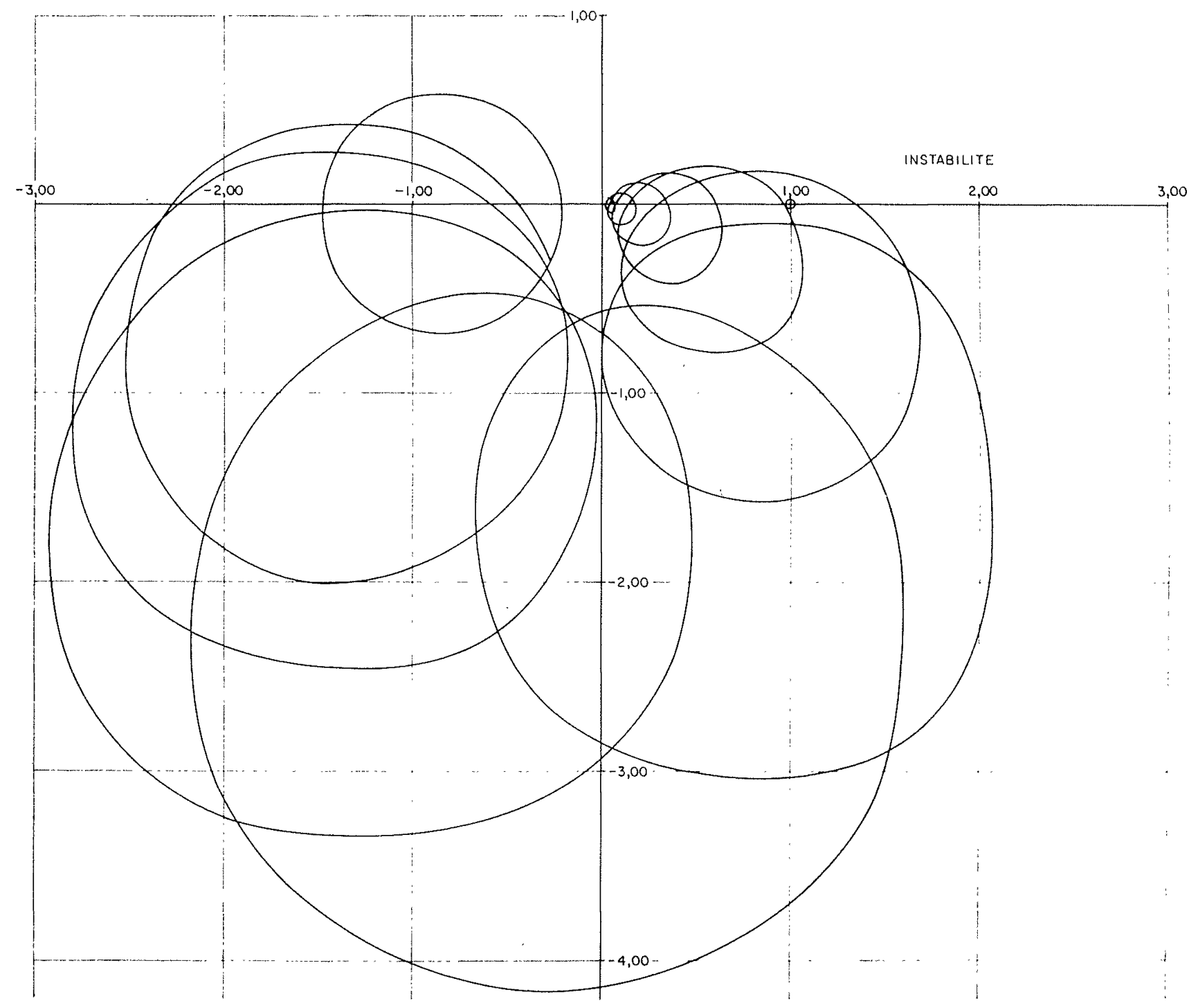

Fig. 5

Régulation d'une vanne automatique. Diagramme de Nyquist. 
courbes représentées dans les figures 5 et 6 . On constate que les courbes obtenues sont loin d'avoir une forme simple et que le travail néces- saire à leur détermination précise ne se justifie que si l'on dispose d'une machine électronique suffisamment rapide.

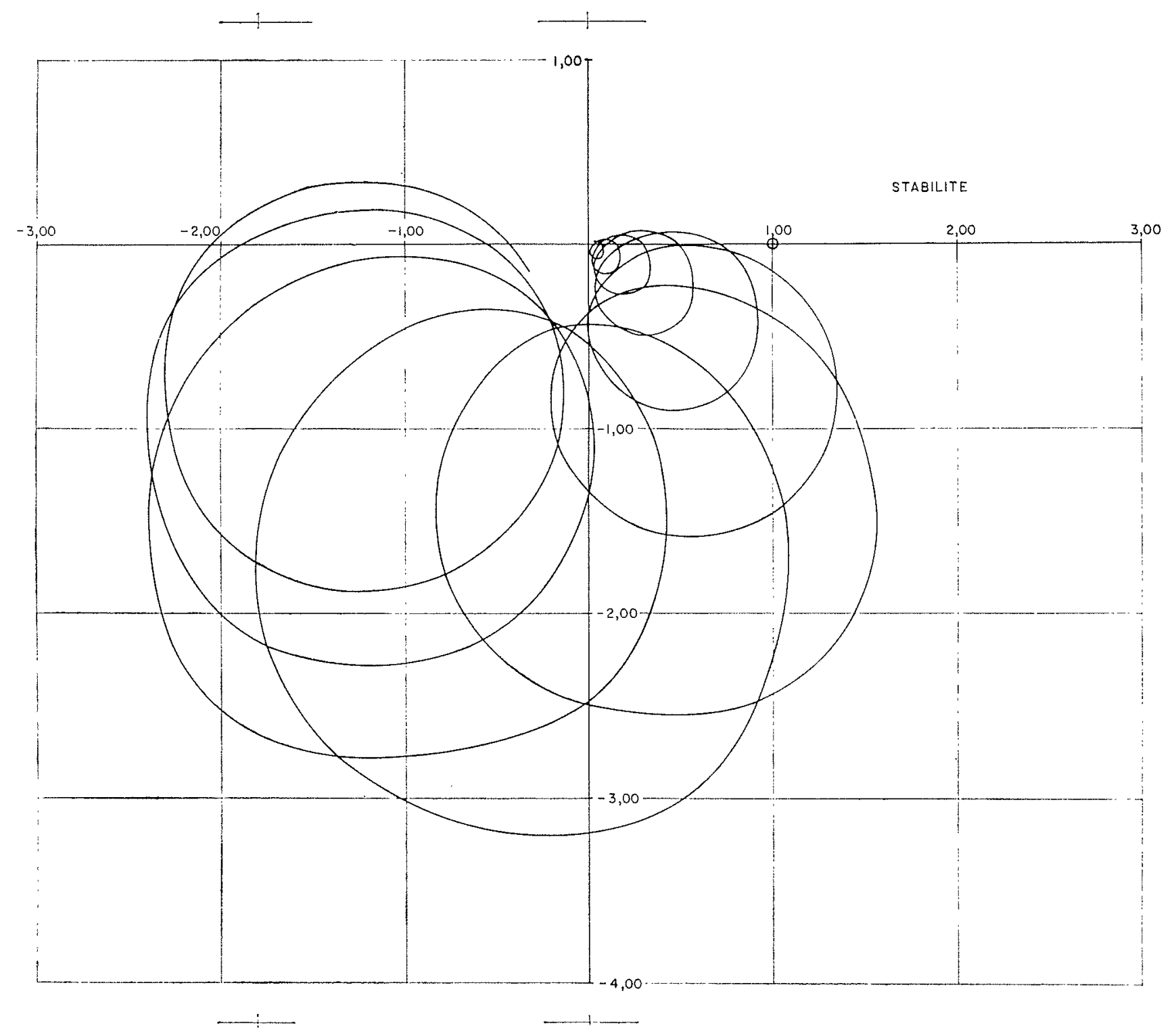

FIG. 6

Régulation d'une vanne automatique.

Diagramme de Nyquist. 


\section{IV. - CALCUL DE L'ECOULEMENT STATIONNAIRE DANS LES GALERIES QUI PEUVENT ETRE TANTOT EN CHARGE, TANTOT A SURFACE LIBRE}

Les programmes que nous avons mis au point pour le calcul des ondes dans les canaux ne prévoient pas le cas des galeries pouvant fonctionner en charge. Le problème de l'écoulement dans les galeries pouvant se mettre en charge semble a priori présenter des difficultés considérables. En réalité, en surmontant les galeries par des fentes de faible largeur qui fonctionnent en quelques sorte comme piézomètres, on est immédiatement ramené au cas d'un écoulement à sur- face libre qui ne diffère que de façon insignifiante de l'écoulement localement en charge. Un calcul effectué pour un cas schématique nous a montré que l'épaisseur de la fente devait être choisie très faible pour qu'on obtienne une reproduction suffisamment fidèle des phénomènes. En outre, le procédé aux différences finies utilisć doit être implicite dans le sens donné à ce terme par Richtmyer.

\section{I S C U S I O N}

Président: M. Remenieras

M. le Président remercie M. Preissmans et ouvre la discussion.

M. Nougaro demande si la méthode proposée par M. Preissmann peut être appliquée à l'étude de la superposition d'intumescences résultant du « pompage 》 de turbines. Cette condition est schématisée par une vanne coupant à intervalle régulier une partie du débit.

M. Preissmann confirme que c'est le même principe mais que la régulation doit, probablement, être un peu différente car il s'agit de régler le nombre de tours. C'est l'application du diagramme de Nyquist. Pour un canal, les formes étudiées sont du type $\Sigma \mathrm{P}^{h}\left(e^{\lambda^{p}}\right)$ au lieu d'être de la forme $P_{1(p)} / P_{2(p)}$. C'est pourquoi les courbes trouvées ont des formes extrêmement compliquées.
M. Thirriot demande à M. Preissmane, s'il a trailé la méthode dont il a parlé à la fin de son exposé et s'il l'a comparée avec des résultats expérimentaux.

M. Preissmann répond qu'i] n'a trailé qu'un cas schématique par cette méthode. Le calcul a été fait pour voir si les résultats semblaient acceptables et on a conclu qu'il fallait prendre une fente extrememont étroite pour obtenir des résultats normaux. Il y a un certain nombre de précautions à prendre dans la définition précise de la section. Il a été prévu d'étudier sur machine électronique le cas de l'usine de Wettingen qui a fait l'objet d'un essai sur modèle réduit.

M. Premsinan pense, d'autre part, que ces cas neuvent ètre calculés sans difficulté.

M. le Président remercie à nouveau M. Pressisman pour son exposé. 